\title{
Modern Computational Techniques for Environmental Data; Application to the Global Ozone Layer
}

\author{
Costas Varotsos* \\ University of Athens, Department of Applied Physics, \\ Bldg. Phys. 5, Panepistimiopolis, GR-157 84 Athens, GR \\ covar@phys.uoa.gr \\ * Currently with the University of Maryland, Department of Meteorology, \\ 3417 Computer and Space Science Bldg., College Park, MD 20742 \\ covar@atmos. umd.edu
}

\begin{abstract}
The physics laws, which govern the atmospheric phenomena, are mostly non-linear and therefore the application of the conventional Fourier spectral analysis on the time series of the atmospheric quantities reveals that these are usually non-stationary. Quite often these non-stationarities conceals the existing correlations and therefore new analytical techniques capable to eliminate non-stationarities in the data should be employed. The most recent analytical methods used along these lines are the wavelet techniques and the detrended fluctuation analysis. Much attention has been paid recently to the latter technique, which has already proved its usefulness in a large variety of complex systems. As a paradigm, the detrended fluctuation analysis is applied to the column ozone data. Specifically the zonally and globally averaged column ozone observations conducted by ground-based (1964-2004) and satellite-borne (1979-2003) instrumentation are employed to detect long-range correlations in column ozone time series. The results show that column ozone fluctuations exhibit persistent long-range power-law correlations for all time lags between 4 months - 11 years.
\end{abstract}

\section{Introduction}

Trends in total ozone content (TOZ) are caused by external effects and they are usually supposed to have a smooth and monotonous or slowly oscillating behavior. Therefore, for the reliable detection of long-range correlations, it is essential to distinguish trends from the long-range fluctuations intrinsic in the data. Usually, the short-range correlations are described by the autocorrelation function, which declines exponentially with a certain decay time. For the long-range correlations, however, the autocorrelation function declines as a power-law. However, the direct calculation of the autocorrelation function is usually not appropriate due to noise superimposed on the collected data and due to underlying trends of unknown origin. The detrended fluctuation analysis (DFA), which will be discussed later, is a well established method for determining the scaling behavior of noisy data in the presence of trends without knowing their origin and shape. 
Very recently, Varotsos [2005] showed that the amplitudes of large TOZ fluctuations (in seasons of enhanced ozone depletion) obey a power-law scaling. This means that correlations between these points decrease according to a power law and are therefore scale-invariant. He also suggested that the Arctic and Antarctic TOZ fluctuations exhibit persistent long-range correlations for all time lags between 4 days - 2.5 years. This means that TOZ fluctuations at different times are correlated and the corresponding correlation function decays much slower than the exponential decay, i.e. a power-law decay. In other words persistence refers to the "memory" or internal correlation within the TOZ time series. For example, there is a tendency an increase in TOZ to be followed by another increase in TOZ at a different time. Furthermore he demonstrated the crucial role of the planetary waves to the scaling dynamics of TOZ over the high latitudes in both hemispheres, since the elimination of the TOZ longterm trend leads to persistent (antipersistent) long-range power-law correlations for time lags shorter (longer) than 10 days. It is worth noting here that a series is persistent if adjacent values are positively correlated, whereas a series is antipersistent if adjacent values are inversely correlated.

To reach to the aforementioned conclusions Varotsos [2005] employed the DFA method, which as mentioned above, allows the detection of long-range power-law correlations in a time-series with noise that often can mask true correlations. In this respect, due to the fact that the physics laws, which govern the atmospheric phenomena, are mostly non-linear, the application of the conventional Fourier spectral analysis on the time series of the atmospheric quantities reveals that these are usually non-stationary (the correlation functions are not invariant under time translation) (Chen et al. 2002, and references therein). Quite often these nonstationarities (e.g. trends and cycles) conceals the existing correlations (or other intrinsic properties) and therefore new analytical techniques capable to eliminate nonstationarities in the data should be employed (Hu et al. 2001).

The most recent methods used along these lines are the wavelet techniques (e.g. Koscielny-Bunde et al. 1998) and the DFA that introduced by Peng et al. (1994). Much attention has been paid recently to the latter technique, which has already proved its usefulness in a large variety of complex systems, for example, in southern oscillation index, in turbulence, in biology, in financial analysis and in other selforganizing critical systems (e.g. Ausloos and Ivanova, 2001; Weber, and Talkner, 2001; Chen et al. 2002; Varotsos et al. 2003ab; Collette and Ausloos 2004).

The present paper examines the time scaling of the TOZ fluctuations over the tropical and mid-latitudinal zones of both hemispheres and globally, thus contributing to the attempt for a selected choice between the proposed climate models for a projection of the TOZ levels in the future, taking into account the feedback between climate change and the ozone layer.

\section{The Time Scaling and Correlations of the TOZ Fluctuations}

The zonally and globally averaged TOZ observations performed by ground-based (1964-2004) and satellite-borne (1979-2003) instrumentation is used in order to 
efficiently search for time scaling, by adopting, however, a data analysis technique, which is not debatable due to the non-stationarity of the data. Thus, to study the temporal correlations of TOZ fluctuations the method of DFA with acceptable error bars is herewith used. This method stems from random walk theory, and permits the detection of intrinsic self-similarity in non-stationary time series (Talkner and Weber 2000). Therefore, this method has the advantage of avoiding seasonal-like trends and non-stationarity effects. According to DFA method, the time series is first integrated and then it is divided into boxes of equal length, $\Delta t$. In each box, a least squares line (or polynomial curve of order $l$, DFA- $l$ ) is then fitted, in order to detrend the integrated time series by subtracting the locally fitted trend in each box. The rootmean-square (rms) fluctuations $F_{d}(\Delta t)$ of this integrated and detrended time series is calculated over all time scales (box sizes). More specifically, the detrended fluctuation function $F(\tau)$ is calculated as follows (Kantelhardt et al. 2002):

$$
F^{2}(\tau)=\frac{1}{\tau} \sum_{t=k \tau+1}^{(k+1) \tau}[y(t)-z(t)]^{2}, k=0,1,2, \ldots,\left(\frac{N}{\tau}-1\right)
$$

where $z(t)=a t+b$ is the linear least-square fit to the $\tau$ data points contained into a class.

For scaling dynamics, the averaged $F^{2}(\tau)$ over the $N / \tau$ intervals with length $\tau$ is expected to obey a power-law, notably:

$$
\left\langle F^{2}(\tau)\right\rangle \sim \tau^{2 \alpha}
$$

and the power spectrum function scales with $1 / f^{\beta}$, with $\beta=2 \alpha-1$.

An exponent $\alpha \neq 1 / 2$ in a certain range of $\tau$ values implies the existence of longrange correlations in that time interval as, for example, in fractional Brownian motion, while $\alpha=1 / 2$ corresponds to the classical random walk (white noise). If $0<\alpha<0.5$, power-law anticorrelations are present (antipersistence). If $0.5<\alpha \leq 1.0$, then $0<\beta$ $\leq 1$, and persistent long-range power-law correlations prevail; the case $\beta=1 \quad(\alpha=1)$ corresponds to the so-called $1 / f$ noise. In addition, when $1<\alpha<1.5$, then long-range correlations are again present (but are stronger than in the previous case); the value $\alpha$ $=1.5$ corresponds to the Brownian noise (e.g., Talkner and Weber, 2000).

It is worth noting that since the time series is first summed the noise level due to imperfect observations is reduced.

It has recently been recognized ( $\mathrm{Hu}$ et al, 2001) that the existence of long-term trends in a time series may influence the results of the correlation analysis. Therefore, the effects of TOZ trends have to be distinguished from TOZ intrinsic fluctuations. To this end, before applying scaling analysis to the TOZ time-series, all TOZ data were deseasonalized and detrended.

In Figure 1, a log-log plot of the function $F_{d}=\sqrt{\left\langle F^{2}(\tau)\right\rangle}$ is shown, by employing the DFA-1 to the deaseasonalised and detrended monthly mean values of the groundbased TOZ values, during $1964-2003$, over the belt $90^{\circ} \mathrm{S}-90^{\circ} \mathrm{N}$. Since $\alpha=1.1( \pm 0.13)$ 
for the interval time ranging from about 4 months to 11 years long-range correlations are present. This suggests persistent long-range power-law correlations in global TOZ fluctuations. This persistence suggests that an anomaly in global TOZ in one time frame continues into the next.

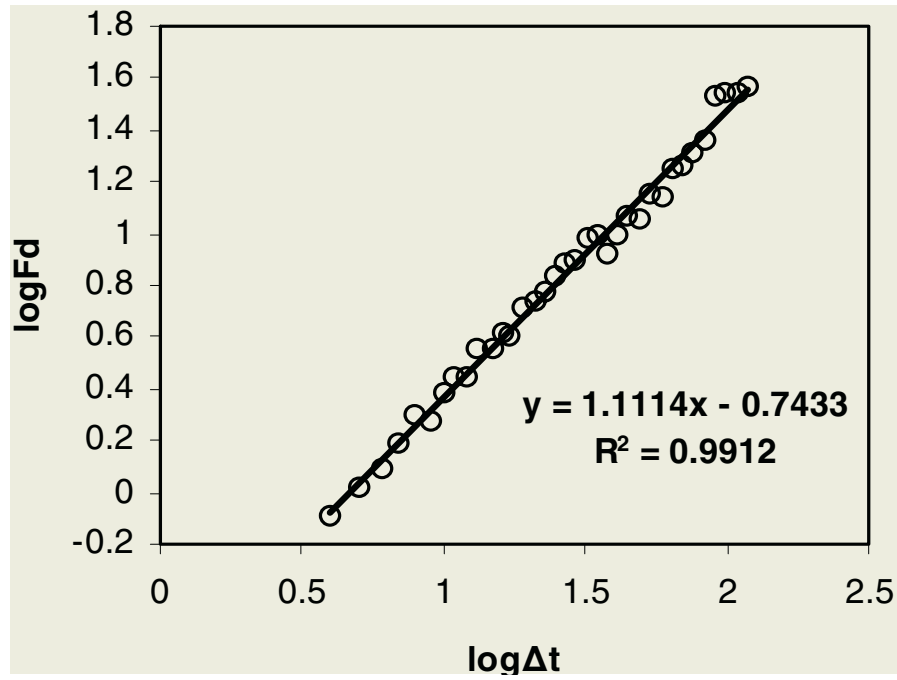

Fig. 1. DFA-function in log-log plot for the deseasonalised and detrended TOZ, during 19642003 , over the belt $90^{\circ} \mathrm{S}-90^{\circ} \mathrm{N}$

In the following, the temporal correlations of the deaseasonalised and detrended monthly mean values of TOZ are also examined for the belt $25^{\circ} \mathrm{S}-25^{\circ} \mathrm{N}$ during 1964 2003. The result obtained from the application of the DFA-1 to the aforementioned TOZ values over tropics is presented at Figure 2. The finding drawn from this figure $(\alpha=1.1 \pm 0.11)$ is that TOZ over tropics exhibits persistent long-range power-law correlations for the interval time ranging from about 4 months to 11 years.

It is worth noting that similar to the above-discussed results are also found by applying the DFA-1 method to the monthly zonal mean V8 TOZ values over tropics and globally.

We now turn to the extra-tropics and specifically to the ground-based TOZ data for the latitude belt $25^{\circ} \mathrm{N}-60^{\circ} \mathrm{N}$ during $1964-2003$. The application of the DFA-1 method to these deseasonalised and detrended monthly mean values of TOZ reveals that longrange power-law correlations exist for all time scales (fig.3a). Since $\alpha_{1}=1.2 \pm 0.13$ (for time scales shorter than 2 years) the long-range correlations in TOZ exhibit "stronger memory" (the process forgets more slowly its past behavior) compared to that of $\alpha_{2}=0.6 \pm 0.09$ (for time scales longer than 2 years). Higher persistence implies a stronger correlation between successive data points. It is also worth noting that "memory" or correlations exist at all time scales over which the power law is valid. 
Finally, the DFA-1 method is also applied on the deseasonalised and detrended monthly mean values of TOZ in the latitude belt $25^{\circ} \mathrm{S}-60^{\circ} \mathrm{S}$ during $1964-2003$. The results obtained are depicted in Fig.3b, where persistency of TOZ fluctuations is observed. In particular, for time scales shorter (longer) than 2 years $\alpha_{1}=1.1 \pm 0.11\left(\alpha_{2}\right.$ $=0.6 \pm 0.07)$.

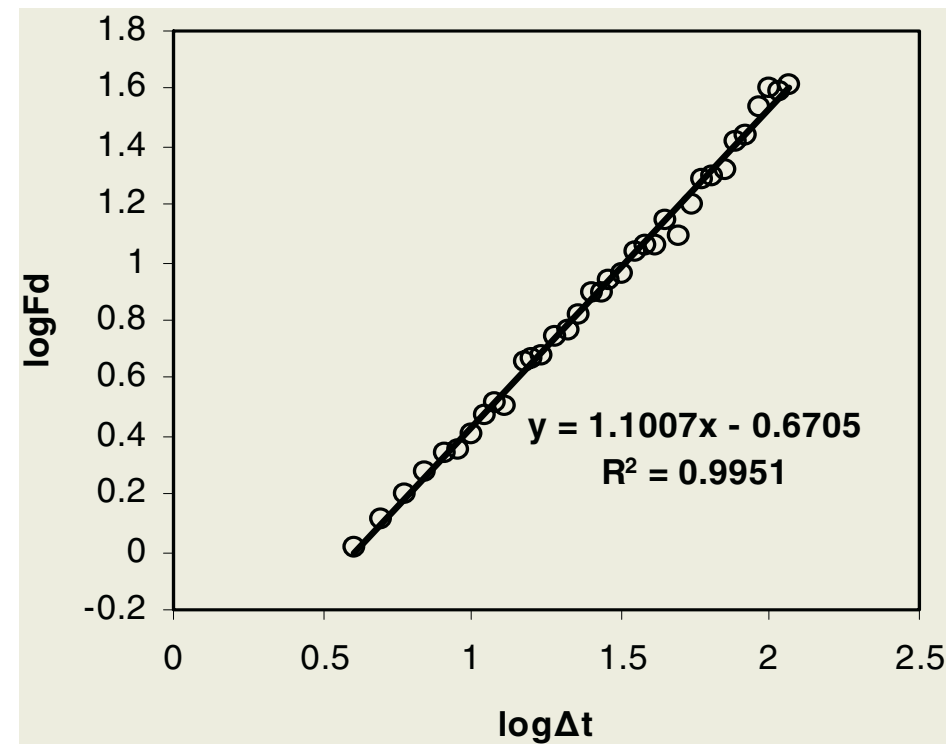

Fig. 2. DFA-function in log-log plot for the deseasonalised and detrended TOZ during 19642003 , over the belt $25^{\circ} \mathrm{S}-25^{\circ} \mathrm{N}$

It is worth mentioning that similar to the above-discussed results for the TOZ variability over extra-tropics are also found by applying the DFA-1 method to the monthly zonal mean V8 TOZ values.

The results obtained clearly show that the monthly mean column ozone fluctuations over tropics, extra-tropics and globally exhibit persistent long-range correlations for all time lags between 4 months - 11 years, which correspond to the $1 / \mathrm{f}$ noise. Over extra-tropics, this persistency becomes weaker for time lags between 2-11 years. It is well known that the $1 / \mathrm{f}$ noise is one of the most common features in nature. Superposition of effects giving rise to signals with scale invariant distributions of correlation times, the so-called scale similarity, could be on the basis of the observed behavior. However, a proper explanation for such a behavior is still lacking and for this reason the physical origin of the $1 / \mathrm{f}$ noise is pretty much an open question (Fanchiotti et al, 2004).

The aforementioned findings seem to favor specific models for the description of the ozone depletion and may lead to better predictability on the global TOZ evolution along the lines of nonlinear dynamics. 

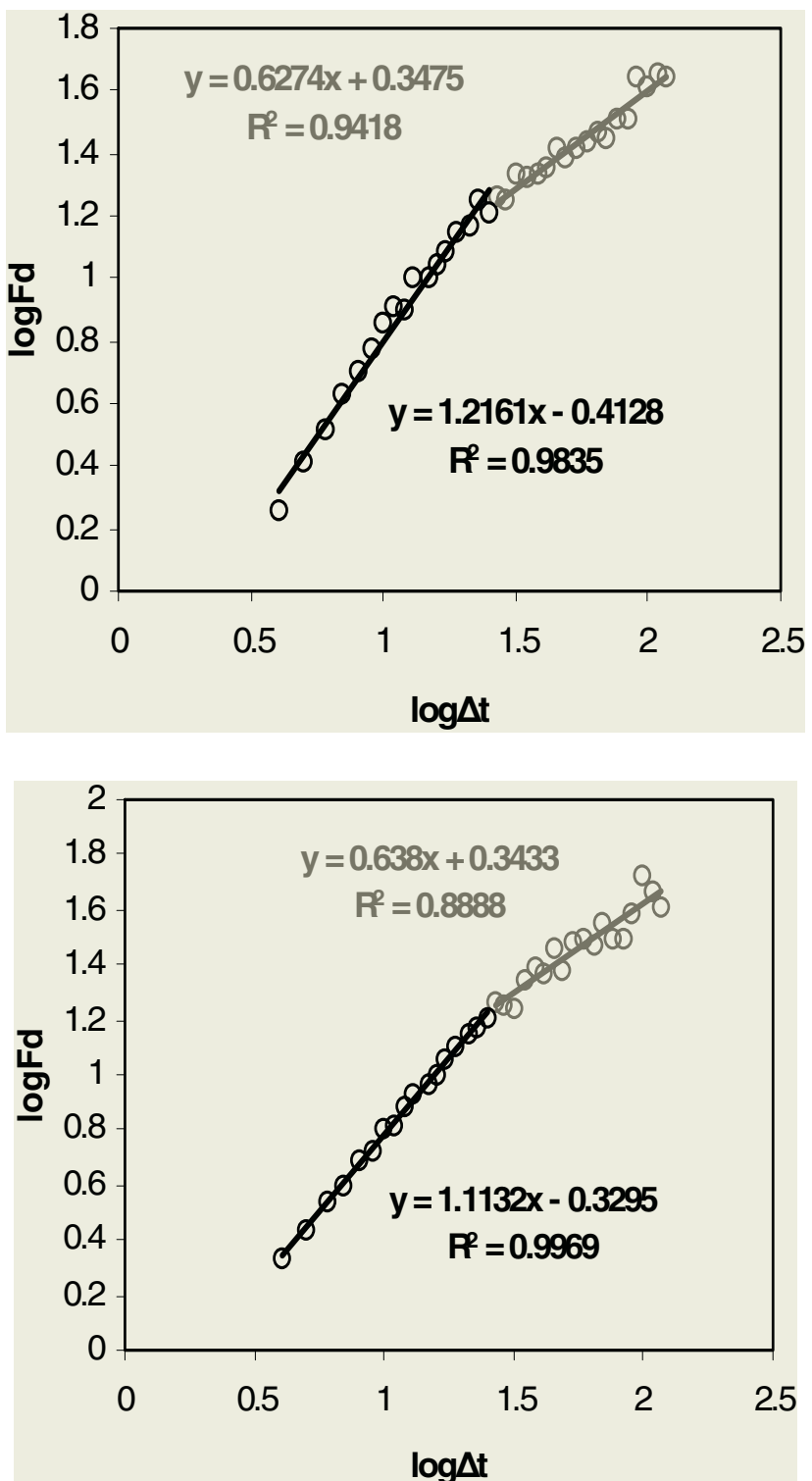

Fig. 3. DFA-function in log-log plot for the deseasonalised and detrended TOZ during 19642003 , over the belt : (a) $25^{\circ} \mathrm{N}-60^{\circ} \mathrm{N}$, and (b) $25^{\circ} \mathrm{S}-60^{\circ} \mathrm{S}$

\section{Conclusions}

The investigation of the existence of the long-range correlations to the zonally and globally averaged column ozone data derived from observations performed by ground-based (1964-2004) and satellite-borne (1979-2003) instrumentation shows the following: 
The monthly mean column ozone fluctuations over tropics, extra-tropics and globally exhibit persistent long-range correlations for all time lags between 4 months - 11 years, which correspond to the 1/f noise. Over extra-tropics, this persistency becomes weaker for time lags between 2-11 years.

The above-mentioned findings demonstrate the nature of specific atmospheric mechanisms that operate and affect the ozone layer in a power law fashion. These could also be a good test of atmospheric chemistry-transport models. Apart from reproducing instantaneous absolute values, model results should also demonstrate the scaling behavior.

\section{Acknowledgments}

TOMS data were produced by the Ozone Processing Team at NASA's Goddard Space Flight Center. The ground-based data are credited to Vitaly Fioletov, Experimental Studies Division, Air Quality Research Meteorological Service of Canada.

\section{References}

Ausloos, M., and K. Ivanova, 2001: Power-law correlations in the southern-oscillation-index fluctuations characterizing El Nino. Phys. Rev. E, 63 (4): art. no. 047201.

Chen, Z., P. C. Ivanov, K. Hu, and H. E. Stanley, 2002: Effect of nonstationarities on detrended fluctuation analysis. Phys. Rev. E, 65 (4), art. no. 041107.

Collette, C., and M. Ausloos, 2004: Scaling analysis and evolution equation of the north atlantic oscillation index fluctuations. ArXiv:nlin.CD/0406068 vl 29 June.

Fanchiotti, H., S.J. Sciutto, C.A. Garcia, and C. Hojuat, 2004: Analysis of sunspot number fluctuations. ArXiv:nlin.AO/0403032 vl 16 March.

Hu, K., P. C.Ivanov, Z. Chen, P. Carpena, and H. E. Stanley, 2001: Effect of trends on detrended fluctuation analysis. Phys. Rev. E 64 (1): art. no. 011114.

Kantelhardt, J.W., S. A. Zschiegner, E. Koscielny-Bunde, S. Havlin, A. Bunde, and H. E. Stanley, 2002: Multifractal detrended fluctuation analysis of nonstationary time series. Physica A 316 (1-4): 87-114.

Koscielny-Bunde, E., A. Bunde, S. Havlin, H. E. Roman, Y. Goldreich, H. J. Schellnhuber, 1998: Indication of a universal persistence law governing atmospheric variability. Phys. Rev. Lett. 81 (3), 729-732.

Peng, C. K., S. V. Buldyrev, S. Havlin, M. Simons, H. E. Stanley, and A. L. Goldberger, 1994: Mosaic organization of DNA nucleotides. Phys. Rev. E 49 (2), 1685-1689.

Talkner, P., and R. O. Weber: 2000: Power spectrum and detrended fluctuation analysis: Application to daily temperatures. Phys. Rev. E 62 (1): 150-160 Part A.

Varotsos, C., 2005: Power-law correlations in column ozone over Antarctica. Int. J. Rem. Sensing (in press).

Varotsos, P. A., N. V. Sarlis, and E. S. Skordas, 2003a: Long-range correlations in the electric signals that precede rupture: Further investigations. Phys. Rev. E, 67, 21109-21121.

---------, ---------, and ----------, 2003b: Attempt to distinguish electric signals of a dichotomous nature. Phys. Rev. E 68 (3): art. no. 031106.

Weber, R. O., and P. Talkner, 2001: Spectra and correlations of climate data from days to decades. J. Geophys. Res., 106, 20131-20144. 